\title{
Tuning of Plasmonic Nanoparticle and Surface Enhanced Wavelength Shifting of a Nanosystem Sensing Using 3-D-FDTD Method
}

\author{
A. Bouali, Shyqyri Haxha, Fathi AbdelMalek, M. Dridi, and Habib Bouchriha
}

\begin{abstract}
In this paper, we have used in-house the 3-D finitedifference time-domain method to analyze a novel design of metallic nanoparticles based on a sensing nanosystem. The proposed structure is composed of two gold-nanocylinders of finite height with varying radii separated by a nanogap. We have demonstrated that tunable plasmonic nanoparticles can be controlled by varying the size of the interparticles separation distance. By engineering the nanogaps, it is shown that a strong enhancement of the electric field is achieved. Our simulations show a pronounced wavelength shift for small nanogaps. In addition, the influence of the refractive index of the surrounding medium is presented.
\end{abstract}

Index Terms-Biosensors, nanophotonics, plasmons, nanoparticles, optical sensors.

\section{INTRODUCTION}

B IOSENSORS at nanoscale regime have found extensive applications and uses in the analysis of various biomolecular interactions (BIA) and detection of chemical and biological analytes in areas such as environmental protection, food safety and medical diagnostics. In last decade, major research has been devoted to designing novel biosensors for various nanoscale applications [1]-[5]. The study of optical properties of nanometric particles offers the opportunity to understand various useful phenomena in physicobiological and physicochemical fields on the nanoscale regime [5]. The metal nanoparticules or surface plasmon polaritons are known as quasiparticles which are generated through coupling between solids and light in quantum systems. These plasmons are the quanta of coupled oscillations of electromagnetic field and electrons in a metal. The electromagnetic field of the plasmon is confined to the vicinity of the metal-dielectric interface. At the interface an enhancement of the field is reached. This is mainly due to the confining nature of the plasmonic waves that propagate along the interface, where its amplitude decays

Manuscript received April 23, 2014; revised June 5, 2014; accepted June 24, 2014. Date of publication June 30, 2014; date of current version July 8, 2014.

A. Bouali and F. AbdelMalek are with the National Institute of Applied Sciences and Technology, Carthage University, Carthage 1054, Tunisia (e-mail: abouali@yahoo.fr; fathi.amalek@gmail.com).

$\mathrm{S}$. Haxha is with the Department of Computer Science and Technology, Bedfordshire University, Bedfordshire LU1 3JU, U.K. (e-mail: shyqyri.haxha@beds.ac.uk).

M. Dridi and H. Bouchriha are with the Quantum Physics and Photonics Group, Department of Physics, El Manar University, Tunis 2092, Tunisia (e-mail: montacer.dridi@utt.fr; habib.bouchriha@fst.rnu.tn).

Color versions of one or more of the figures in this paper are available online at http://ieeexplore.ieee.org.

Digital Object Identifier 10.1109/JQE.2014.2333420 exponentially as the distance increases from the interface. Since the field decays with the distance from the surface, this behavior cannot be detected and observed by far field analysis, unless the evanescent field is manipulated to propagate along a relatively long distance. This technique can be achieved by engineering the surface. The strong confinement of the field results in an extraordinary sensitivity of the plasmonic waves to the surrounding medium. This sensitivity has been used to study the absorbed nanoparticules and colorants on the surface.

The plasmonic nanoparticles have drawn growing attention worldwide recently, due mainly to their unprecedented optical properties related to the surface plasmon resonance [6]. Due to their unique ability to confine the light beyond the diffraction limit, plasmonic nanoparticles promise very exciting opportunities. It was demonstrated that when two plasmonic nanoparticles are brought close to each other, the electromagnetic field is enhanced significantly; this is due to the fact that the energy is confined and concentrated between the two nanoparticles [7]. In such a system, the local excitation of resonant nanoplasmonic particles allows the confinement of energy in small volumes. The enhanced field in these volumes has been used in many applications, including near-field microscopy and nanoscale optical devices [8]. The enhancement of optical near fields can be employed to cover the infrared frequency regions. This leads to a strong enhancement in infrared spectroscopy. Similarly the field enhancement can be used in surface enhanced Raman scattering effects [9].

These optical properties depend strongly on the size and shape of the nanoparticles and are influenced by the surrounding medium. These types of quasi particles find many areas of applications such as subwavelength control of light, light focusing and light confinement. The study of metallic particles is not novel; previously Maxwell had presented the first explanation related to glasses containing metallic particles [10]. Recently, the study of spectroscopic behavior of resonant-coupled particles has attracted the attention of many researchers [11], [12]. They proposed to investigate the tuning of the coupled systems as a function of the geometric configurations such as the separation distance and the polarization direction [13]. Experimental work has been conducted to measure the field variation generated between a gold nanoparticle and a nanowaveguide, where the shifts of the resonant peaks are assigned to the modes related to the separation distance [14]. The field enhancement of 
asymmetric plasmonic nanoparticles have been studied by many world class researchers; Ryan et al., [15] have reported field enhancement induced between a metallic tip and metallic substrate [15]. The enhancement of the field has been investigated in multi-material trimmer nanostructures composed of $\mathrm{Au}$ nanoparticles surrounded by two Ag nanoparticles as a function of the gap between the two Ag nanoparticle and their size. Toroghi et. al. [16] have reported field enhancement factor of around ten times larger than observed in isolated $\mathrm{Au}$ nanoparticles.

In this study, we show that extinction spectra of the coupled nanoparticles exhibit resonance modes located in the visible spectral range. The diffraction and interference of the out coupled waves are investigated by using, in-house, the 3D-Finite Difference Time Domain (3D -FDTD) technique [17], [18]. The FDTD grid is excited using the total-field/scattered field formulation [15] with plane-wave polarized parallel to the $\mathrm{x}$-axis. The source is placed at the cell grid number 4 after the PMLs. We use pulse excitation of a bandwidth around $10 \mathrm{~nm}$. This model implemented in an FDTD algorithm is highly accurate in a wavelength range of $500 \mathrm{~nm}$ to $1200 \mathrm{~nm}$ [17]. The central wavelength is $600 \mathrm{~nm}$. The computation of the optical spectra of the system is performed by considering the spatial parameters $\Delta \mathrm{x}=3 \mathrm{~nm}$ and $\Delta \mathrm{t}=\Delta \mathrm{x} /(2 \mathrm{c})$, the incident light is polarized along the $\mathrm{x}$-axis, where $\mathrm{c}$ represents the speed of light in vacuum. The propagation of the incident plane wave is along the z-axis and the gold nanoparticles are deposited on an anisotropic substrate, where the $x-y$ plane is parallel to the surface of the substrate. The Drude-Lorentz model is used to describe the dispersive properties of the gold nanoparticles.

\section{Numerical Simulation}

The structure under this study is composed of two gold nanoparticles, where the extraordinary transmission measurements can be performed under normal incidence from the bottom. The incident plane wave propagates along the z-axis, and the $x-y$ plane is parallel to the surface of the substrate on which the particles are deposited. The substrate is anisotropic, however the host material is assumed isotropic where each nanoparticle consists of a cylinder of $200 \mathrm{~nm}$ height and varying radius. The anisotropic substrate is a commercial E7 liquid crystal, with ordinary refractive index $n_{0}=1.53$ and extraordinary refractive index $n_{e}=1.74$ [19], [20]. The dimer based nanoparticles separated by the nanogaps are represented in Fig. 1. In order to investigate the performance sensitivity of the proposed nanosystem, several analytes with different refractive indices are tested.

The 3D-FDTD method is employed to calculate the resonance wavelength, evolution of the fields, and the extinction spectra of the nanostructure. This numerical method with a central finite difference of the continuous derivatives of Maxwell equations in leapfrogs and staggered grid is well known and is widely used in electromagnetism and spectroscopy. It should be noted that the proposed structure has dispersive properties; therefore modifications of the iterative equations are required. Hence, in the 3D FDTD calculations, the permittivity of gold is assumed to be a set of DrudeLorentz poles. In its complex form, the dielectric function of

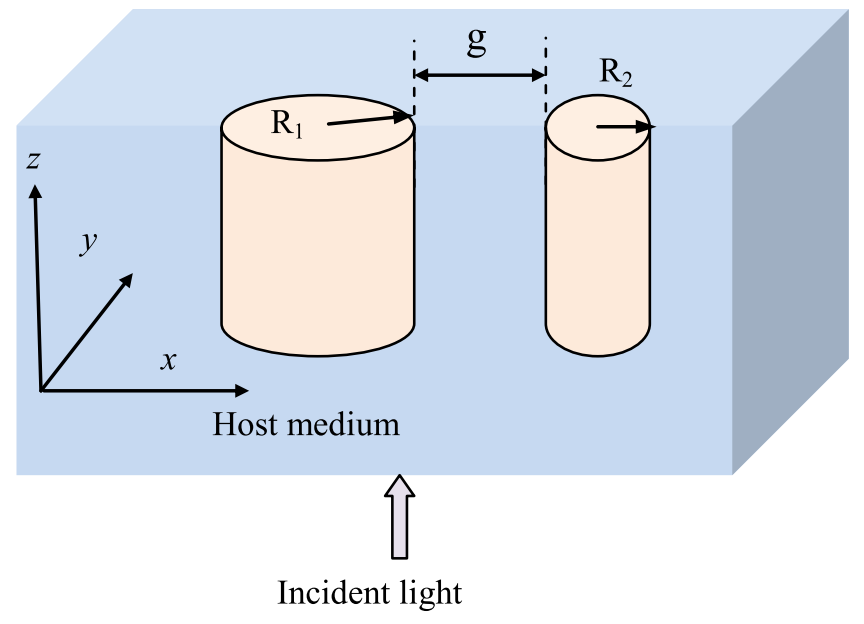

Fig. 1. Nanosystem formed by two gold-nanparticles of radius $R_{1}$ and $R_{2}$ separated by a nanogap, g set in a host medium.

the gold particles reads

$$
\varepsilon_{D L}=\varepsilon_{\infty}-\frac{\omega_{D}^{2}}{\omega\left(\omega+j \gamma_{D}\right)}-\frac{\Delta \varepsilon \times \Omega_{L}^{2}}{\left(\omega^{2}-\Omega_{L}^{2}\right)+j \omega \Gamma_{L}}
$$

where $\omega_{\mathrm{D}}$ stands for the plasma frequency, $\gamma_{\mathrm{D}}$ the damping coefficient, $\Omega_{\mathrm{L}}$ the oscillator strength, $\Delta \varepsilon$ is a weighting factor, and $\Gamma_{\mathrm{L}}$ represents the spectral width of the Lorentz oscillators. In our calculation, the parameters are taken from Ref. [21], where the wavelength range is from $500 \mathrm{~nm}$ to $1200 \mathrm{~nm}$. The calculations of the extinction are performed within the wavelength range, varying from $500 \mathrm{~nm}$ to $1400 \mathrm{~nm}$. The resonant wavelength corresponds to the maximum extinction as a function of lambda. The signals are recorded at each time step, and monitors are set at the top of the nanocylinders (output). The structure is periodic in $x-y$ directions, therefore we use the periodic boundary conditions in these directions, whereas absorbing boundary conditions are deployed in $\mathrm{z}$ direction. This allows simulating the extension of the structure by placing two uniaxial Perfectly Matched Layer (PML) regions at the top and the bottom in order to absorb the waves. In this case, the relation between electric flux and electric field is tonsorial. The relation between the electric flux, $\vec{D}$ and $\vec{E}$ is given by a tonsorial expression

$$
\left(\begin{array}{c}
D_{x} \\
D_{y} \\
D_{z}
\end{array}\right)=\left(\begin{array}{ccc}
\varepsilon_{0} \varepsilon_{x x} & \varepsilon_{0} \varepsilon_{x y} & \varepsilon_{0} \varepsilon_{x z} \\
\varepsilon_{0} \varepsilon_{y x} & \varepsilon_{0} \varepsilon_{y y} & \varepsilon_{0} \varepsilon_{y z} \\
\varepsilon_{0} \varepsilon_{z x} & \varepsilon_{0} \varepsilon_{z y} & \varepsilon_{0} \varepsilon_{z z}
\end{array}\right)\left(\begin{array}{c}
E_{x} \\
E_{y} \\
E_{z}
\end{array}\right)
$$

From equation (2), the evaluation of $\mathrm{D}_{\mathrm{z}}$ depends on the $\mathrm{x}$ and $\mathrm{y}$ components of $\mathrm{D}$. We need to determine these components at the same location [22]:

$$
\begin{aligned}
E_{z} \mid \begin{array}{c}
n+1 \\
i, j, k+1 / 2
\end{array}= & c_{z x} D_{x} \mid \begin{array}{c}
n+1 \\
i, j, k+1 / 2
\end{array} \\
& +c_{z y} D_{y} \mid \begin{array}{c}
n+1 \\
i, j, k+1 / 2
\end{array} \\
& +c_{z z} D_{z} \mid \begin{array}{c}
n+1 \\
i, j, k+1 / 2
\end{array}
\end{aligned}
$$

In the FDTD procedure, the components $\mathrm{D}_{\mathrm{x}}$ and $\mathrm{D}_{\mathrm{y}}$ should fit the grid and in order to achieve this, we use the following 


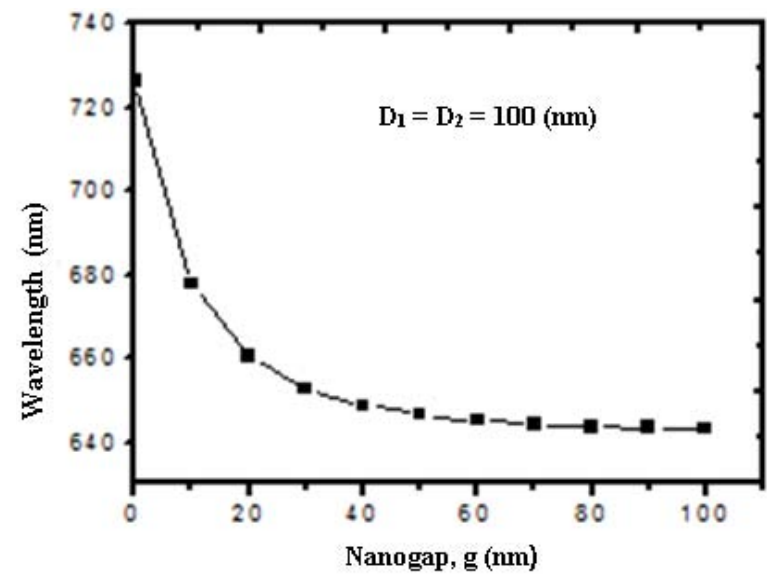

Fig. 2. Wavelength variation with $g$ when the diameters of nanoparticles are $\mathrm{D}_{1}=\mathrm{D}_{2}=100 \mathrm{~nm}$.

approximation [23]:

$$
\begin{aligned}
\left.D_{x}\right|_{i i, j, k+1 / 2}= & \frac{1}{4}\left(\left.D_{x}\right|_{i+1 / 2, j, k}+\left.D_{x}\right|_{i-1 / 2, j, k-1 / 2}\right) \\
& +\frac{1}{4}\left(\left.D_{x}\right|_{i+1 / 2, j, k+1}+\left.D_{x}\right|_{i-1 / 2, j, k+1}\right)
\end{aligned}
$$

Similar procedure we apply for $\mathrm{D}_{\mathrm{y}}, \mathrm{E}_{\mathrm{x}}$ and $\mathrm{E}_{\mathrm{y}}$.

\section{RESULTS AND Discussions}

We investigate the nanosystem symmetry effect on the wavelength shifts of the proposed nanosystem model consisting of two gold-nanocylinders with a height of $200 \mathrm{~nm}$ and varying radii. The periodicity of the array is $200 \mathrm{~nm}$. The proposed nanosystem structure is depicted in Fig. 1. We calculate the resonant frequency as a function of the nanogap, g, which is defined as the separation distance between the two gold nanoparticles in the cylindrical shape. The result is illustrated in Fig. 2, where the radii of the nanoparticles, $R_{1}$, and $R_{2}$ are equal to $50 \mathrm{~nm}$.

Fig. 2 shows that the resonant wavelength decreases rapidly with $\mathrm{g}$ in a range varying from $\mathrm{g}=0 \mathrm{~nm}$ to $\mathrm{g}=50 \mathrm{~nm}$ which is the maximum value of $g$ for which the resonant wavelength decreases rapidly $\left(\mathrm{g}_{\text {limt }}\right)$. As the nanogap exceeds $\mathrm{g}_{\text {limt }}$, the wavelength starts to flatten and reaches the saturation in the gap range $80 \mathrm{~nm}$ to $100 \mathrm{~nm}$. In this region the nanosystem is not sensitive to the changes of the interparticle distance. The value $\mathrm{g}_{\text {limt }}$ represents the maximum value of $\mathrm{g}$ for which the resonant wavelength decreases rapidly before reaching the saturation.

Moreover, from Fig. 2 one may notice that resonant wavelength shifts to $\lambda_{\text {resonant }}=730 \mathrm{~nm}$ when the gap size is about $10 \mathrm{~nm}$. This means that the dipolar modes along the axis of the cylinders oscillate and emit radiation that is shifted towards the red region of the spectrum. When we increase the gap, the resonance wavelength shifts to a smaller wavelength region and a nonlinear blue shift is observed within the gap region from $20 \mathrm{~nm}$ to $60 \mathrm{~nm}$. When the gap exceeds glimit $50 \mathrm{~nm}$, the wavelength starts to flatten and reaches the saturation in the gap range $80 \mathrm{~nm}$ to $100 \mathrm{~nm}$. At a small nanogap the resonance of the plasmon is strong, which leads to a larger shift of the resonance. This agrees well with the fact that when

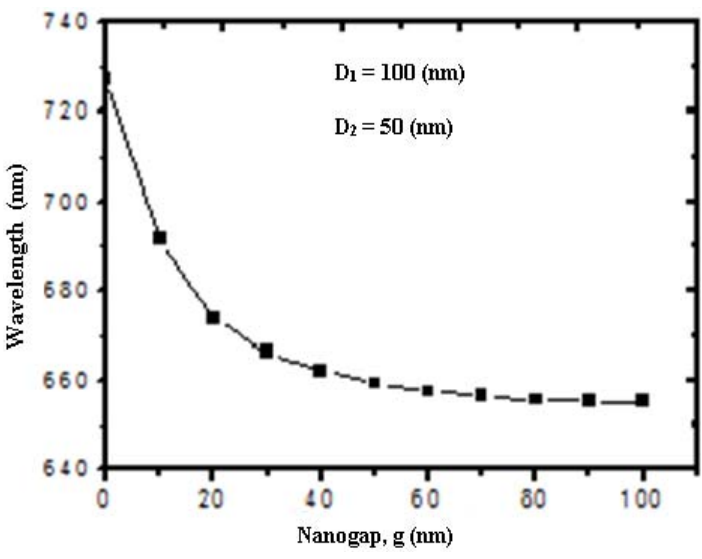

Fig. 3. Wavelength variation with $g$ when $D_{1}=100 \mathrm{~nm}$ and $D_{2}=50 \mathrm{~nm}$.

placing two particles closely separated by a critical distance, a strong electrodynamic interaction will take place; this kind of interaction is near-field in nature. Consequently, a larger shift of resonance is achieved. Fig. 2 also shows that the localized plasmon resonance varies slightly when the nanogap exceeds $50 \mathrm{~nm}$. The localized surface plasmon resonance peak redshifts and increases in intensity. The localized surface plasmon is a solution to the equation $\operatorname{Re}(\varepsilon(\omega))=-2 \varepsilon_{1}$ where $\varepsilon(\omega)$ and $\varepsilon_{1}$ satisfies the dipole moment equation (see equation 5).

By shinning a metallic nanoparticle with an external field, a dipole can be created due to the induced charges. Based on this understanding, the external field $\mathrm{E}_{0}$ creates a dipole moment on the particle that can be expressed as:

$$
P=\varepsilon_{1} \alpha(\omega) E_{0}
$$

where the polarizability $\alpha(\omega)$ is given as follows

$$
\alpha(\omega)=\frac{\varepsilon(\omega)-\varepsilon_{1}}{\varepsilon(\omega)+2 \varepsilon_{1}}
$$

where $\mathrm{P}$ is the dipole moment induced in the nanoparticle system and $\varepsilon_{1}$ and $\varepsilon(\omega)$ are the permittivities of the host medium and the nanoparticles, respectively.

In order to meet requirements for different applications, nanoengineering approaches were developed such as tailoring the size of the nanoparticles. Breaking the symmetry of the nanosystem by changing the radius of one particle and keeping the other one constant has a substantial effect on wavelength tuning. Consequently, we decrease the diameter of one particle to $50 \mathrm{~nm}$ and keep the other one fixed at $100 \mathrm{~nm}$. It is worth noting that breaking the symmetry in such structures has crucial influence on the plasmon coupling to incident radiation. In line with this, pronounced dipole, quadrupole, octupole and fano resonances have been observed in individual cross structures such as Verellen et. al. [24]. Furthermore, the nanocross geometry proves to be a useful building block for coherently coupled plasmonic dimers. Then we calculate the resonant wavelength of the nanosystem, the results of which are illustrated in Fig. 3. One can see that compared to Fig. 2, the wavelength is slightly reduced however; the general trend behavior of the curves (in Fig. 2 and Fig. 3) remains unchanged. Also, from Fig. 3 we notice that the resonant wavelength depends strongly on the interparticle distance 
when $\mathrm{g}$ is smaller than $20 \mathrm{~nm}$. Furthermore, Fig. 3 shows how this dependence is observed for nanogaps larger than $20 \mathrm{~nm}$. It is important to note that the break of symmetry (nanoparticles with different diameters) enhances the resonant wavelength shift. In order to support the interpretation and explain the trade-off between nanogap $g$ and resonant wavelength, we calculate the field intensity normalized by its intensity at the input section for various values of the nanogap, $g$. The intensity, I, is defined as the square modulus of the total electric field, $I=\left|E_{t o t}\right|^{2}$.

We decrease the diameter of one nanoparticle to be $\mathrm{D}_{2}=50 \mathrm{~nm}$ and keep nanoparticle $\mathrm{D}_{1}$ fixed to $100 \mathrm{~nm}$, the wavelength shift is calculated and reported in Fig. 3. This figure shows that for a small $\mathrm{g}$ (less than $20 \mathrm{~nm}$ ), the wavelength shifts to a large value of the spectrum. The maximum wavelength reaches $750 \mathrm{~nm}$, meaning that for a small $\mathrm{g}$ the shift tends to the infra-red region. It should be noted that in this case, for large values of $\mathrm{g}$, the minimum in wavelength shift is less than obtained in the previous case when $\mathrm{D}_{1}=\mathrm{D}_{2}=100 \mathrm{~nm}$, shown in Fig. 2. It should be noted that the surface plasmons exhibit high frequencies, as high as $100 \mathrm{THz}$, however the qualify factor (Q) is smaller than the dielectric counterpart.

In order to analyze and better understand the effect of the nanoparticle size on the wavelength shifts, determining the snapshots of the field distribution is essential. Fig. 4 (a) shows the snapshots of the field in the $\mathrm{x}-\mathrm{y}$ plane when $\mathrm{g}=90 \mathrm{~nm}$. It should be noted that these calculations were performed at the resonant wavelength when the radius of the first nanoparticle is $50 \mathrm{~nm}$ and the radius of the second particle is $25 \mathrm{~nm}$. It is clear from this figure that the field intensity is strongly localized in close proximity of each nanoparticle. The spots of the field are well confined in the nanocylinders. However, their intensities decrease evanescently as the distance between them increases.

Fig. 4 (b) demonstrates calculated results of the field intensity when the nanogap $\mathrm{g}$ is decreased to $70 \mathrm{~nm}$. The field intensity tends to become more confined in the nanoparticle with a smaller radius $\mathrm{g}=70 \mathrm{~nm}$ compared to $\mathrm{g}=90 \mathrm{~nm}$ in Fig. 4 (a). Therefore, we keep reducing the nanogap g to $50 \mathrm{~nm}$. Simulated results for $\mathrm{g}=50 \mathrm{~nm}$ are shown in Fig. 4 (c). It is clear from this figure that the field intensity is enhanced significantly compared to the case where $\mathrm{g}=90 \mathrm{~nm}$. Also, Fig. 4 (c) shows that the field intensity remains localized in the interparticle distance region. From these simulations, we can anticipate that the field intensity/enhancement observations are of high interest and the interparticle distance is the key parameter that can control the field intensity/enhancement.

Motivated by the pronounced effects of the nanogap on the field enhancement, we decided to further reduce the nanogap g between the two gold nanoparticles. In Fig. 4 (d) we illustrate the snapshot of the electric field in the $x-y$ plane when the nanogap, g, is $40 \mathrm{~nm}$. This figure indicates that the field becomes more enhanced compared to cases shown in Figs. 4 (a), (b), (c).

Fig. 5 (a) shows the field intensity, when $\mathrm{g}=30 \mathrm{~nm}$. It is evident that a higher enhancement factor of the electric field is achieved compared to previous cases obtained when $\mathrm{g}=90 \mathrm{~nm}, 70 \mathrm{~nm}$ and $50 \mathrm{~nm}$, respectively. From these

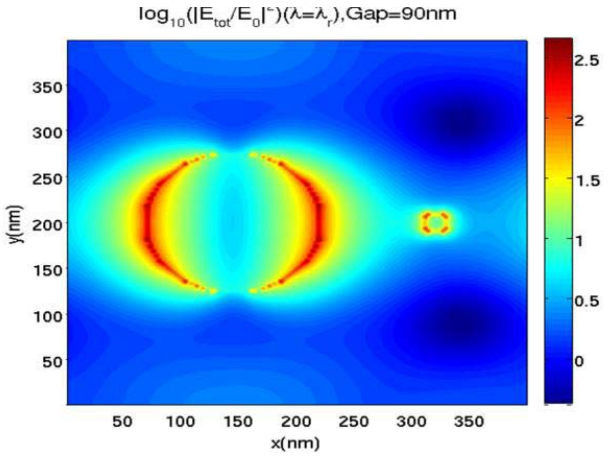

(a)

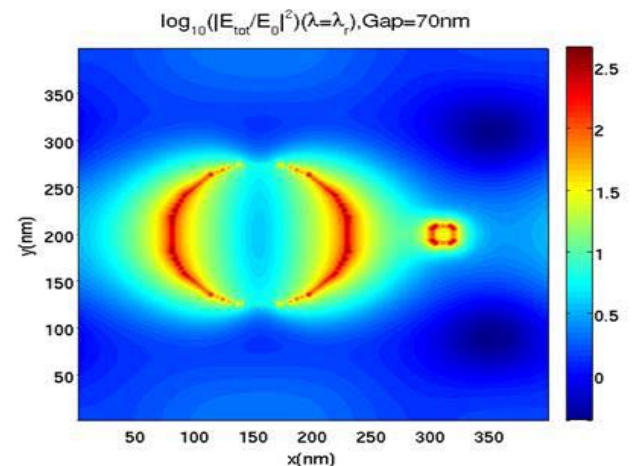

(b)

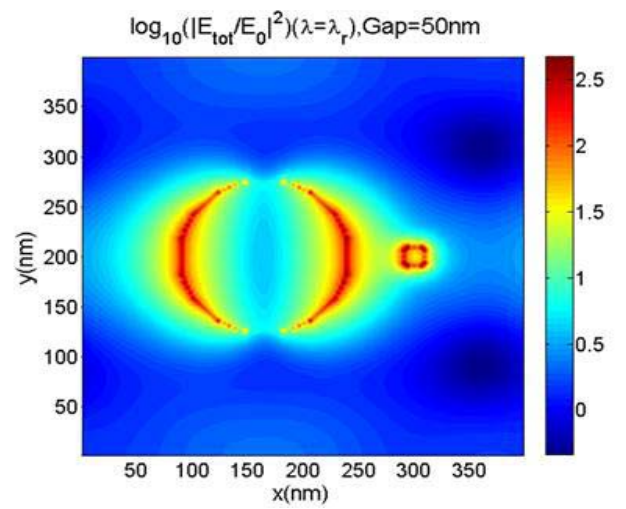

(c)

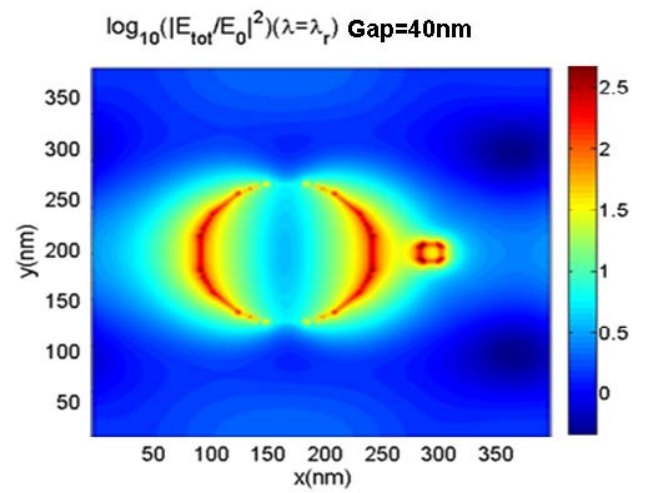

(d)

Fig. 4. Snapshot of the electric field when the nanogap, (a) $g=90 \mathrm{~nm}$ and (b) $\mathrm{g}=70 \mathrm{~nm}$. (c) $\mathrm{g}=50 \mathrm{~nm}$ and (d) $\mathrm{g}=40 \mathrm{~nm}$.

simulated results it is evident that the enhancement of the electric field in the nanoparticle system can be manipulated and controlled by varying the interparticle distance. Therefore, we further reduce $\mathrm{g}$ and make it as small as $10 \mathrm{~nm}$. 


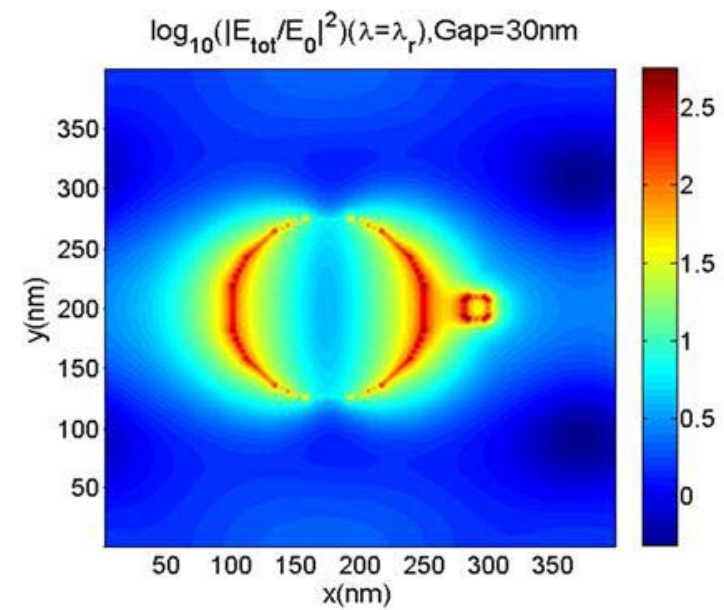

(a)

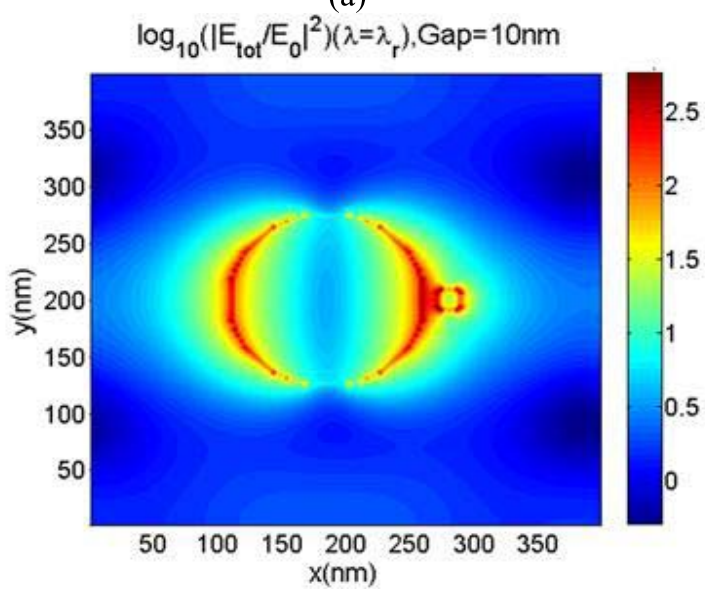

(b)

Fig. 5. Snapshot of the electric field when the nanogap, (a) $\mathrm{g}=30 \mathrm{~nm}$ and (b) $\mathrm{g}=10 \mathrm{~nm}$.

In Fig. 5 (b) we report the snapshot of the field in $x-y$ plane when $\mathrm{g}=10 \mathrm{~nm}$. To have more details on the snapshot of the field when $\mathrm{g}$ is as small as $10 \mathrm{~nm}$, the discretization step should be smaller than used in previous cases, however the limitation in space computing remains difficult. It is evident that strong localized field intensity is present at the center and around the nanoparticle. It is obvious that when the radius of the nanoparticle is small, the dipole moment increases and oscillating dipole behavior is present. Under the external excitation, an induced dipolar moment appears inside each nanoparticle and since the nanogap is small, the moments interact with each other and overlap. This results in a strong enhancement of the electric field around the nanoparticle. Also, this figure indicates a significant enhancement of the field intensity at the edge of the nanoparticle showing a coupling of the energy between the latter and immediate environment present in the nanogap. Thus, the metal nanoparticles enhance the electric fields around themselves due to the surface plasmon. This effect is of great importance for sensing applications where the field distribution is the main key factor since the detection of molecules depends on their interaction with localized field.

It should be noted that these figures (Fig. 4 and Fig 5) represent the snapshots of the time domain response of the plasmonic modes. For the convenience of the space,

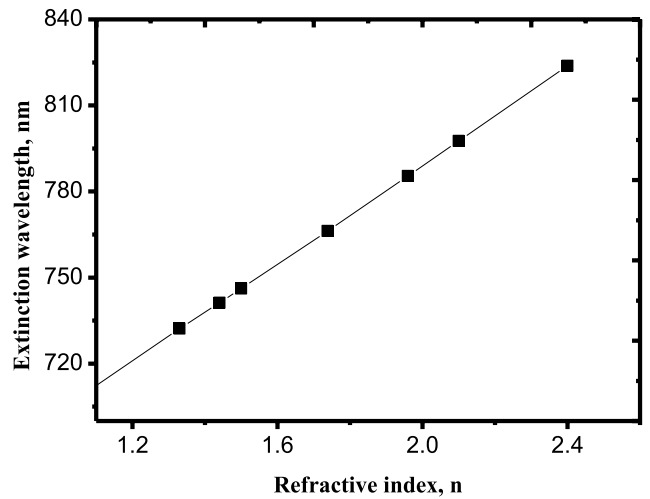

Fig. 6. Extinction wavelength against the refractive index, $\mathrm{n}$.

we consider two nanoparticles, the structure is periodic in the $\mathrm{x}-\mathrm{y}$ directions where the computational volume is truncated with periodic boundary conditions. The periodic boundary conditions are straightforward to implement as the wave front has no time delay and the stability criteria is unchanged. However, in the $\mathrm{z}$ direction the computational volume is truncated with absorbing boundary conditions to simulate the extension of the lattice to infinity. This truncation is performed by placing two Uniaxial Perfect Matched Layer (UPML) regions at the top and the bottom to absorb the waves.

\section{Sensitivity Properties}

Next, we analyze the sensitivity properties of the nanosystem to determine the optimum environmental medium exhibiting the desired sensitivity. The sensitivity is defined as the wavelength shift corresponding to a unit variation of the refractive index; analytically is defined as [2]:

$$
\mathrm{S}=\mathrm{d} \lambda / \mathrm{dn}
$$

We keep the distance between gold nanoparticles, $g$, to the fixed value of $10 \mathrm{~nm}$, and then we set the nanosystem in different mediums with various refractive indices to investigate its sensitivity performance. The following mediums with refractive indices varying from 1 to 2.4 , including water, are replicated. From our simulations we notice that the response increases with the refractive index, n. Also, the wavelength shifts to the infra-red region as the refractive index $\mathrm{n}$ increases. It is clear from our simulations that observed that the proposed nanosystem can be employed to detect substances with high refractive indices in the infra-red region.

In Fig. 6, we illustrate the variation of the extinction wavelength against the refractive index. It is evident from this figure that the dependence is linear with a positive slope. This indicates that the proposed nanosystem is very sensitive to the refractive index changes.

Motivated by the desire to understand these observations, we calculated the wavelength shift $\Delta \lambda$ when the diameters of the nanoparticles are kept fixed to $D_{1}=100 \mathrm{~nm}$ and $D_{2}=50 \mathrm{~nm}$ and the result is shown in Fig. 7. It is clear from this figure that $\Delta \lambda$ varies linearly with the refractive index difference. This result confirms the fact that for small nanogaps, the system is very sensitive to small changes of the refractive index, leading to an accurate detection of substances. It is 


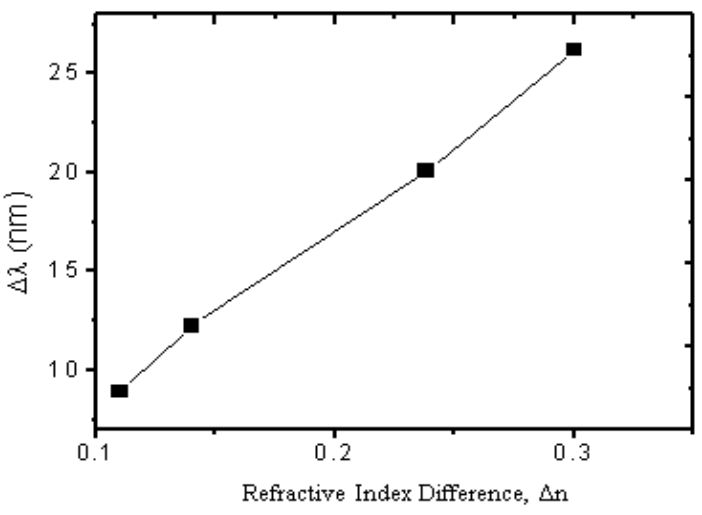

Fig. 7. Wavelength shift $\Delta \lambda$ as a function of $\Delta \mathrm{n}$.

important to notice that in this nanoscale configuration, the proposed nanosystem can be used to detect cancer diseases and toxic substances [25], [26].

\section{CONCLUSION}

A novel design of a metallic gold nanoparticle based nanosystem is proposed and simulated by using in-house 3D FDTD numerical method. The spectral tunability and electric field enhancements are investigated by breaking the symmetry of the nanosystem. By reducing the radius of one of the nanoparticles, a distinct infra-red shift is observed. Effects of the nanogap on the enhancement of the electric field and on the localized surface plasmon resonances are reported in this study. It is demonstrated that a strong enhancement of the field intensity is obtained for a nanogap between gold nanoparticles as small as $10 \mathrm{~nm}$. For a small nanogap between the gold nanoparticles a significant enhancement of the electric field is achieved inside the nanoparticle with the small radius. It is shown that energy exchange between the nanoparticles depends strongly on the size of the nanogap. It is also demonstrated that the proposed design is very sensitive to the refractive index of the surrounding medium. Sensors often are limited by their size, sensitivity, and efficiency; in this regard the proposed nanosensing model would provide nanosensing accuracy beyond conventional methods. This nanosystem will enable sensors to be developed and deployed that can detect at the single molecule level; that can sense weak electromagnetic and gravitational fields with unprecedented precision, leading to quantum sensing technologies. The proposed nanosystem sensors will provide new nanoscale models for medical, environmental monitoring and healthcare technologies. Future work is underway to investigate the sensing performance of gold nanoparticle shape geometries and to further reduce the dimensions in the presence of various surrounding mediums.

\section{REFERENCES}

[1] E. K. Akowuah, T. Gorman, S. Haxha, and J. V. Oliver, "Dual channel planar waveguide surface plasmon resonance biosensor for an aqueous environment," Opt. Exp., vol. 18, no. 24, pp. 24412-24422, Nov. 2010.

[2] E. K. Akowuah, T. Gorman, and S. Haxha, "Design and optimization of a novel surface plasmon resonance biosensor based on Otto configuration," Opt. Exp., vol. 17, no. 26, pp. 23511-23521, Dec. 2009.

[3] F. Ouerghi, F. AbdelMalek, S. Haxha, E. K. Akowuah, and H. Ademgil, "Design of multicavities on left-handed photonic-crystal-based chemical sensors," IEEE J. Lightw. Technol., vol. 30, no. 20, pp. 3288-3293, Oct. 15, 2012.
[4] E. K. Akowuah, T. Gorman, H. Ademgil, S. Haxha, G. K. Robinson, and J. V. Oliver, "Numerical analysis of a photonic crystal fiber for biosensing applications," IEEE J. Quantum Electron., vol. 48, no. 11, pp. 1403-1410, Nov. 2012.

[5] Y. Hennequin, D. G. A. L. Aarts, J. O. Indekev, H. N. W. Lekkerkerker, and D. Bonn, "Fluctuation forces and wetting layers in colloid-polymer mixtures," Phys. Rev. Lett., vol. 100, no. 17, p. 178305, May 2008.

[6] J. Ctyroky, F. AbdelMalek, K. Usbeck, and W. Eck, "Modeling of surface plasmon resonance waveguide sensors with Bragg gratings," Opt. Quantum Electron., vol. 31, nos. 9-10, pp. 927-947, Oct. 1999.

[7] P. K. Arawind, A. Nitzan, and H. Metiu, "The interaction between electromagnetic resonances and its role in spectroscopic studies of molecules adsorbed on colloidal particles or metal spheres," Surf. Sci. vol. 110, no. 1, pp. 189-204, Sep. 1981.

[8] Z. B. Wang, B. S. Lukyanchuk, M. H. Hong, Y. Lin, and T. C. Chong, "Energy flow around a small particle investigated by classical Mie theory," Phys. Rev. B, vol. 70, no. 3, p. 035418, Jul. 2004.

[9] J. P. Kottmann, O. J. F. Martin, B. Hecht, and D. W. Pohl, "Resonant optical antennas," Science, vol. 308, no. 5728, pp. 1607-1609, Jun. 2005.

[10] J. Garnett, "Colours in metal glasses and in metallic films," Philosoph. Trans. Roy. Soc. London A, vol. 203, pp. 385-420, Jan. 1904.

[11] W. S. Chang, L. S. Slaughter, B. P. Khanal, P. Manna, E. R. Zubarev, and S. Link, "One-dimensional coupling of gold nanoparticle plasmons in self-assembled ring superstructures," Nano lett., vol. 9 no. 3, pp. 1152-1157, Feb. 2009.

[12] M. Dridi and A. Vial, "Modeling of metallic nanostructures embedded in liquid crystals: Application to the tuning of their plasmon resonance," Opt. Lett., vol. 34, no. 17, pp. 2652-2654, Sep. 2009.

[13] Y. M. You, C. L. Du, Y. Ma, J. Kasin, T. Yu, and Z. X. Shen, "Nearfield coupling effect on far-field inelastic scattering imaging of $\mathrm{Au}$ nanoparticles," Nanotechnology, vol. 19, no. 39, p. 395705, Oct. 2008.

[14] A. Pack, W. Grill, and R. Wannemcher, "Apertureless near-field optical microscopyof metallic nanoparticles," Ultramicroscopy, vol. 94, no. 2, pp. 109-123, Feb. 2003.

[15] R. M. Roth et al., "Resonant-plasmon field enhancement from asymmetrically illuminated conical metallic-probe tips," Opt. Exp., vol. 14, no. 7, pp. 2921-2931, Apr. 2006.

[16] S. Toroghi, C. Lumdee, and P. G. Kik, "Cascaded plasmon resonances multi-material nanoparticle trimmers for extreme field enhancement," Proc. SPIE, vol. 8809, p. 88091M, Sep. 2013.

[17] A. Taflove and S. Hagness, "Computational electrodynamics," The Finite Difference Time Domain, 3rd ed. Boston, MA, USA: Artech House, May 2005.

[18] F. AbdelMalek, W. Belhadj, and H. Bouchriha, "FDTD study of subwavelength imaging by a photonic crystal slab," Photon. Nanostruct. Fundam. Appl., vol. 3, no. 1, pp. 19-24, Aug. 2005.

[19] W. Dickson, G. A. Wurtz, P. R. Evans, R. J. Pollard, and A. V. Zayats, "Electronically controlled surface plasmon dispersion and optical transmission through metallic hole arrays using liquid crystal," Nano Lett., vol. 8, no. 1, pp. 281-286, Dec. 2008.

[20] I. Abdulhalim, "Surface plasmon TE and TM waves at the anisotropic film-metal interface," J. Opt. A, Pure Appl. Opt., vol. 11, no. 1, p. 015002, Jan. 2009.

[21] A. Vial, A. S. Grimault, D. Macias, D. Barchiesi, and M. L. de la Chapelle, "Improved analytical fit of gold dispersion: Application to the modeling of extinction spectra with a finite-difference time-domain method," Phys. Rev. B, vol. 71, no. 8, p. 085416, Feb. 2005.

[22] M. Dridi and A. Vial, "FDTD modeling of gold nanoparticle pair in a nematic liquid crystal cell," J. Phys. Chem. C, vol. 114, no. 21, pp. 9541-9545, May 2010.

[23] L. Dou and A. R. Sebak, "3D FDTD method for arbitrary anisotropic materials," Microw. Opt. Technol. Lett., vol. 48, no. 10, pp. 2083-2090, Oct. 2006.

[24] N. Verellen, P. Van Dorpe, D. Vercruysse, G. A. E. Vandenbosch, and V. V. Moshchalkov, "Dark and bright localized surface plasmons in nanocrosses," Opt. Exp., vol. 19, no. 12, pp. 11034-11051, Jun. 2011.

[25] D. B. Chithrani, "Nanoparticles for improved therapeutics and imaging in cancer therapy," Recent Patents Nanotechnol., vol. 4, no. 3, pp. 171-180, Nov. 2010.

[26] E. Boisselier and D. Astruc, "Gold nanoparticles in nanomedicine: Preparations, imaging, diagnostics, therapies and toxicity," Chem. Soc. Rev., vol. 38, no. 6, pp. 1759-1782, Apr. 2009. 
A. Bouali is currently pursuing the Ph.D. degree in employing metallic nanoparticles to design new components such as ultracompact sensors. He is involved in a joint project between the Faculty of Science, Tunis El Manar University, Tunis, Tunisia, and Aix-Marseille University, Marseille, France.

Shyqyri Haxha (SM'06) received the M.Sc. and Ph.D. degrees from City University London, London, U.K., in 2000 and 2004, respectively. He has received several world class industrial E.M.B.A. and M.B.A. diplomas In 2014, he joined the Computer Science and Technology, University of Bradfordshire, Luton, U.K., as a Senior Lecturer in Electronic Engineering. Prior to this post, he was a Lecturer in Optic Communication with the Broadband and Wireless Communications Group, Department Electronics, Kent University, Canterbury, U.K., as a Lecturer. His current research interests include nanophotonics, photonic crystal devices, metamaterials, biosensors, photonic crystal fibers, surface plasmon polaritons, high-speed modulators, optical MIMO, optical code division multiple access, and optical MIMO and wireless communication systems. He is a member of the Optical Society of America and the Institution of Engineering and Technology (IET), London. $\mathrm{He}$ is currently leading the IET accreditation of B.Eng. and M.Sc. programs at the Computer Science and Technology, University of Bedfordshire. He was a recipient the SIM Postgraduate Award from The Worshipful Company of Scientific Instrument Makers, Cambridge, U.K., for his highly successful contribution in research in 2003. He has been a Keynote Speaker of numerous world class conferences. He is listed in Who's Who in the World.

Fathi AbdelMalek received the Ph.D. degree from the Ecole Centrale de Lyon, Lyon, France, in 1999. He was a Research Associate and a Research Fellow at various U.S. and U.K. universities, where he carried out research on sensors grating for telecommunication, nanoplasmonic, and photonics. $\mathrm{He}$ is currently a Professor with the Department of Physics and Engineering, National Institute of Applied Sciences and Technology, Tunis, Tunisia. He is also with the College of Optical Sciences, University of Arizona, Tucson, AZ, USA. His current research interests include nanophotonics, nanoplasmonic crystals, metamaterials, microstructured fibers, developing novel approaches to subcellular imaging, quantum dots, biosensors, and numerical techniques such as finite-difference time domain and finite-element methods. He is involved in the design of integrated nanophotonic devices.
M. Dridi received the Ph.D. degree from the Université de Technologie de Troyes, Troyes, France, in 2010, focused on liquid crystals. He is currently a Post-Doctoral Researcher in Plasmonics. His expertise is on the modeling of metallic nanostructures and tuning of their plasmon resonance for applications in sensing.

Habib Bouchriha received the M.Sc. degree in physics, the Ph.D. degree, and the Doctorate of States degree from the University of Paris, Paris, France, in 1970, 1972, and 1978, respectively. In 1980, he was the first President of the Tunisian Society of Physics. Since 1978, he has been a Professor of Physics with the University of Tunis, Tunis, Tunisia. He is the Head of the Advanced Materials, Quantum Physics, and Photonics Groups, Quantum Physics, and Photonics Laboratory at the Faculty of Sciences, University of Tunis. His research interest is focused on molecular crystals, physics of polymer, photonic crystals, quantum wells, magnetic resonance, and lightemitting diodes. He was a recipient of the Medal of Tunisian Education Merit in 1994, and the Distinguished Rammal Medal from the French Physical Society and Ecole Normale Superieure de Paris Foundation in 1995. 